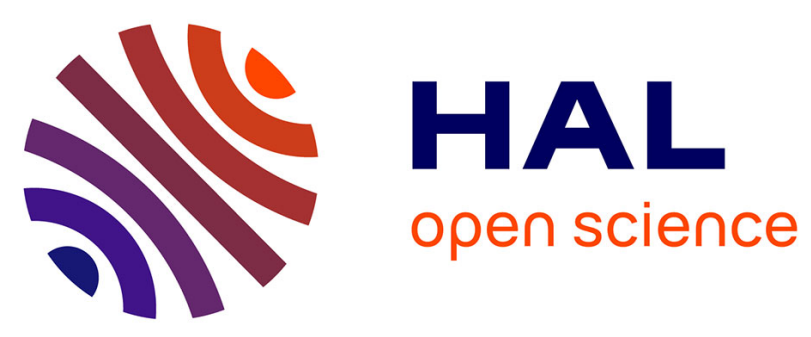

\title{
Near-Field Scanning Optical Microscope Combined with Digital Holography for Three-Dimensional Electromagnetic Field Reconstruction
}

Nancy Rahbany, Ignacio Izeddin, Valentina Krachmalnicoff, Rémi Carminati, Gilles Tessier, Yannick de Wilde

\section{To cite this version:}

Nancy Rahbany, Ignacio Izeddin, Valentina Krachmalnicoff, Rémi Carminati, Gilles Tessier, et al.. Near-Field Scanning Optical Microscope Combined with Digital Holography for ThreeDimensional Electromagnetic Field Reconstruction. Vasily Astratov. Label-Free Super-Resolution Microscopy, Springer, Cham, pp.113-136, 2019, Biological and Medical Physics, Biomedical Engineering, 10.1007/978-3-030-21722-8_5 . hal-02358251

\section{HAL Id: hal-02358251 \\ https://hal.science/hal-02358251}

Submitted on 11 Nov 2019

HAL is a multi-disciplinary open access archive for the deposit and dissemination of scientific research documents, whether they are published or not. The documents may come from teaching and research institutions in France or abroad, or from public or private research centers.
L'archive ouverte pluridisciplinaire HAL, est destinée au dépôt et à la diffusion de documents scientifiques de niveau recherche, publiés ou non, émanant des établissements d'enseignement et de recherche français ou étrangers, des laboratoires publics ou privés. 


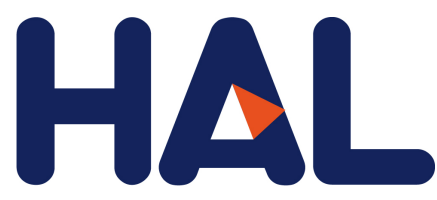

archives-ouvertes

\section{Near-Field Scanning Optical Microscope Combined with Digital Holography for Three-Dimensional Electromagnetic Field Reconstruction}

Ignacio Izzedin, Remi Carminati, Nancy Rahbany, Ignacio Izeddin, Valentina Krachmalnicoff, Rémi Carminati, Gilles Tessier, Yannick de Wilde

\section{To cite this version:}

Ignacio Izzedin, Remi Carminati, Nancy Rahbany, Ignacio Izeddin, Valentina Krachmalnicoff, et al.. Near-Field Scanning Optical Microscope Combined with Digital Holography for Three-Dimensional Electromagnetic Field Reconstruction. Label-Free Super-Resolution Microscopy Editor: Vasily Astratov Springer, Cham, pp.113-136, 2019, 10.1007/978-3-030-21722-8_5 . hal-02358251

\section{HAL Id: hal-02358251 \\ https://hal.archives-ouvertes.fr/hal-02358251}

Submitted on 11 Nov 2019

HAL is a multi-disciplinary open access archive for the deposit and dissemination of scientific research documents, whether they are published or not. The documents may come from teaching and research institutions in France or abroad, or from public or private research centers.
L'archive ouverte pluridisciplinaire HAL, est destinée au dépôt et à la diffusion de documents scientifiques de niveau recherche, publiés ou non, émanant des établissements d'enseignement et de recherche français ou étrangers, des laboratoires publics ou privés. 


\title{
Chapter
}

\section{Near-field scanning optical microscope combined with digital holography for three-dimensional electromagnetic field reconstruction}

\author{
Nancy Rahbany, Ignacio Izzedin, Valentina Krachmalnicoff, Rémi Carminati, Gilles Tessier, Yannick De \\ Wilde
}

\begin{abstract}
Near-field scanning optical microscopy (NSOM) has proven to be a very powerful imaging technique that allows overcoming the diffraction limit and obtaining information on a scale much smaller than what can be achieved by classical optical imaging techniques. This is achieved using nanosized probes that are placed in close proximity to the sample surface, and thus allow the detection of evanescent waves that contain important information about the properties of the sample on a subwavelength scale. In particular, some aperture-based probes use a nanometer-sized hole to locally illuminate the sample. The far-field radiation of such probes is essential to their imaging properties, but cannot be easily estimated since it highly depends on the environment with which it interacts. In this chapter, we tackle this problem by introducing a microscopy method based on full-field off-axis digital holography that allows us to study in details the three-dimensional electromagnetic field scattered by a NSOM probe in different environments. We start by describing the NSOM and holography techniques independently, and continue by highlighting the advantage of combining both methods. We present a comparative study of the reconstructed light from a NSOM tip located in free space or coupled to transparent and plasmonic media. While far-field methods, such as back focal plane imaging, can be used to infer the directionality of angular radiation patterns, the advantage of our technique is that a single hologram contains information on both the amplitude and phase of the scattered light, allowing to reverse numerically the propagation of the electromagnetic field towards the source. We also present Finite Difference Time Domain (FDTD) simulations to model the radiation of the NSOM tip as a superposition of a magnetic and an electric dipole. We finally propose some promising applications that could be performed with this combined NSOMholography technique.
\end{abstract}

\subsection{Introduction to Near-field scanning optical microscopy (NSOM)}

Near-field Scanning Optical Microscopy (NSOM) is an important super-resolution imaging technique which is used to overcome the diffraction limit and study light-matter interaction at a subwavelength scale [1].

N. Rahbany, I. Izzedin, V. Krachmalnicoff, R. Carminati, Y. De Wilde

ESPCI Paris, PSL University, CNRS, Institut Langevin

1 rue Jussieu, F-75005, Paris, France

e-mail: yannick.dewilde@espci.fr

G. Tessier

Sorbonne Université, CNRS, INSERM, Institut de la Vision

17 Rue Moreau, 75012 Paris, France

e-mail: gilles.tessier@sorbonne-universite.fr

E. Hanssen (ed.), Cellular Imaging, Biological and Medical Physics,

Biomedical Engineering, https://doi.org/10.1007/978-3-319-68997-5_1 


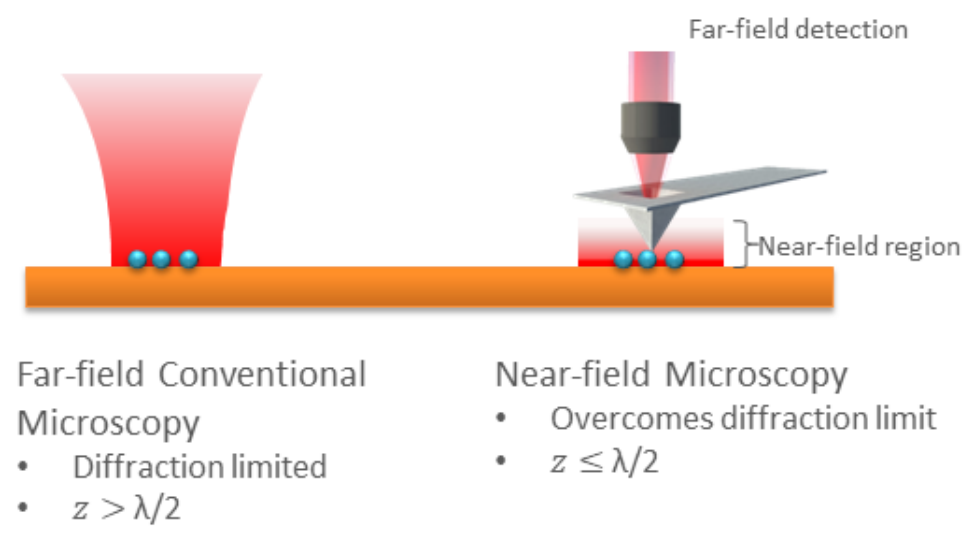

Fig. 1.1 Schematic of the advantages of SNOM compared to conventional far-field microscopy.

Since the high frequency spatial components of the electromagnetic (EM) field are contained in the near field and decay exponentially with the distance from the sample, they are entirely lost when using far-field optical microscopy techniques. Therefore, the idea of nearfield microscopy is to collect the information contained in the near field and make it measurable with traditional far-field detection tools. Scattering-type NSOM uses a scanning probe, which is typically a tip with a sub- $\lambda$ sized scatterer at its extremity, to measure the evanescent waves that are confined in the near field. The evanescent signal is scattered from the near field and is converted to a far-field propagating wave which is directed to a detector measuring its intensity. Then, the sample is scanned with respect to the tip to obtain a complete image of the scanned area point by point. Probing the near field allows to overcome the diffraction limit, which is why NSOM has proven to be a powerful "super-resolution" technique. The method allows one indeed to perform sub- $\lambda$ imaging, or to detect purely evanescent fields such as surface plasmon polaritons (SPPs). A schematic is shown in Fig 1.1. The resolution of an image obtained by NSOM depends on three parameters: the distance between adjacent points in the scanned area, the size of the tip, and the distance between the tip and the sample. Evidently, the highest resolution is obtained for a small tip apex and a small tip-sample distance. Fig 1.2 shows an example of a NSOM image taken from [2], where the propagating SPPs generated by metallic grating couplers are clearly detected.

The history of the NSOM technique dates back to the beginning of the twentieth century, when E. Synge in collaboration with A. Einstein [3,4] came up with two concepts that led to the two main NSOM families known today: the apertureless scattering NSOM, and the aperture NSOM. Experimentally, the first near-field optical microscope functioning in the visible range was developed in $1986[1,5]$.

- Apertureless scattering NSOMs include a diffusing tip or a nanoparticle that scatters the near-field signal into the far-field. In free space, such tips are usually modeled as effective electric dipoles where the electric field component parallel to the tip axis is enhanced [6-8]. 


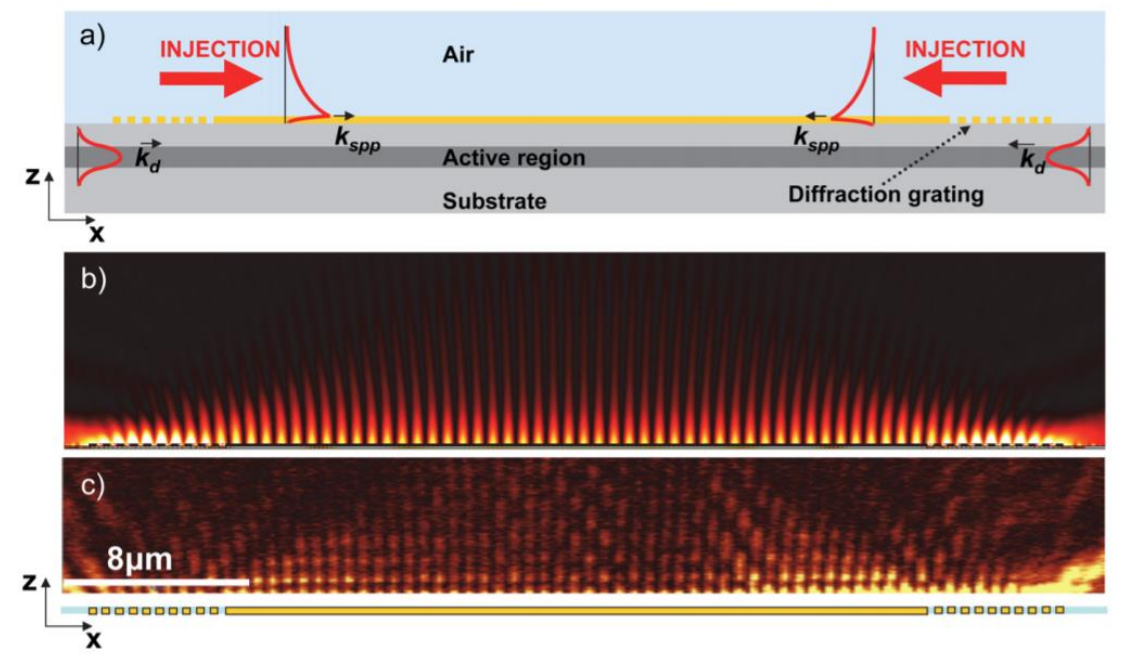

Fig. 1.2 Scattering NSOM measurement: a) Schematic side view of the metallic grating couplers device imaged here. Details about the structure can be found in [9]. The red curves schematically represent the mode profiles of waveguided SPP modes. b) Color-plot of the calculated squared modulus of the electric field in the $\mathrm{x}-\mathrm{z}$ plane (SPP interference pattern). $\mathrm{c}$ ) NSOM measurement in the $x-z$ plane, i.e., NSOM signal as a function of the tip-to-surface distance at several x-positions on the sample. The measurement qualitatively agrees with the simulation in panel b. Figure taken from [2].

- Aperture NSOMs use tips fitted with a nano-aperture. This tip is sometimes made of metal, with a hole at its extremity, or most often a tapered metal-coated optical fiber (except for its end, where the aperture is located). Aperture SNOM tips can therefore be used in two different ways: as a collection aperture to detect the near-field signal, or as a nanoscale source of light through which the sample is illuminated locally. In the latter case, a precise knowledge of the characteristic of this nanosource is essential in order to quantitatively understand images obtained by scanning it above the sample. In particular, the shape and directionality (scattering diagram) of such sources are essential to the imaging properties of such systems, but are poorly known, either for SNOM tips in air, or in contact with a surface.

Aperture SNOM tips were first studied theoretically by Bethe [10] and were modeled as a subwavelength hole in a perfectly conducting plane screen. Its radiation in free-space corresponded to that of a coherent superposition of a magnetic and an electric dipole $[6,11-13]$. However, recent studies have shown that metal-coated hollow pyramidal probes behave solely as tangential magnetic dipoles when placed in the vicinity of metallic nanoantennas [14]. Therefore characterizing NSOM probes is not a straightforward and easy task. Their radiation patterns strongly depend on the type of probe as well as on the environment that they interact with. When such probes are placed close to the surface of a sample, the scattered field is highly affected by multiple reflections and interferences at the surface. This strong coupling between the probe and the surface makes it even more difficult to predict their EM radiation pattern in a given environment $[15,16]$. 
4

In addition to its detection role, a NSOM tip can also be used to excite and launch surface plasmon polaritons on the surface of metallic films [17]. It is important to mention here that the excitation of surface plasmons can also be performed by other techniques such as exciting surface defects [6,17], pointlike dipoles [18], and tunneling electrons [19,20], in addition to classical techniques such as prism or grating couplers. The far-field radiation pattern of leaky surface plasmons launched on metallic thin films by NSOM tips was previously studied using conventional back focal plane imaging techniques [17,21,22]. In such techniques, the intensity of the EM field is accurately measured in the Fourier plane of an optical system $[23,24]$. However, no information about the phase is obtained. In order to fully characterize the scattered EM field in three dimensions, both the intensity and the phase must be calculated. For this reason, we developed a combined NSOM-holography technique to accurately describe the radiation patterns of individual NSOM probes as a function of the local environment. The main advantage of our combined system is that it can directly deliver information about both the amplitude and the phase of the scattered light through the NSOM probe from a single recorded hologram. Then, following the procedure described in section 1.2, we can reconstruct the full three-dimensional scattered field coupled to the environment [25]. It is important to mention that Digital Holographic Microscopy was previously combined with NSOM for the purpose of achieving super-resolution imaging through a disordered scattering medium that is illuminated by subwavelength tips that act as point-like sources [26].

\subsection{Principles of Digital Holography}

Holography is a technique that combines the processes of interference and diffraction to record and reconstruct the amplitude and phase of an electromagnetic field in three-dimensions. It was discovered in 1948 by Denis Gabor [27,28] who received a Nobel Prize in Physics for his work later in 1971. It wasn't until the development of the lasers in 1960 that holography took its place in the optics domain [29].

The advantage of holography over conventional photography techniques is that it contains information about the entire three-dimensional wavefield which is contained in interference patterns. These patterns arise when the wave scattered by the object, or object wave, is illuminated by a reference wave, creating a hologram. This hologram is then illuminated with the reference wave again to obtain the three-dimensional reconstructed image of the electromagnetic field scattered by the object [30-36].

The general holography method is depicted in Fig 1.3. For recording a hologram (Fig 1.3 a, left image), light from the laser is split into a reference beam and an object beam that illuminates the object. Both beams are directed to a detector where they interfere creating a hologram. For the reconstruction process (Fig 1.3 a, right image) of photographic-plate holograms, the developed hologram is illuminated with the reference wave. This creates a virtual image of the three-dimensional reconstructed object at a distance $d$, which is the position where the object had been before. Nowadays, holography is almost exclusively conducted using cameras, and the latter reconstruction step is performed digitally. 
a)

$\underline{\text { Recording }}$

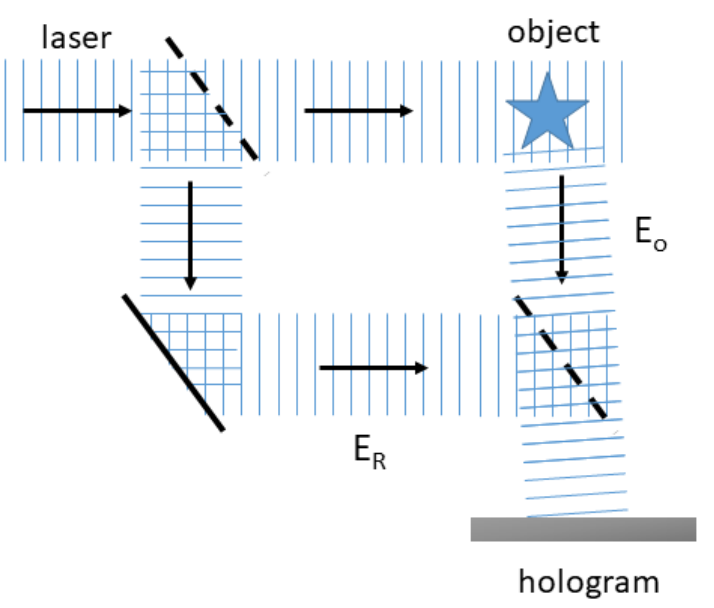

Reconstruction

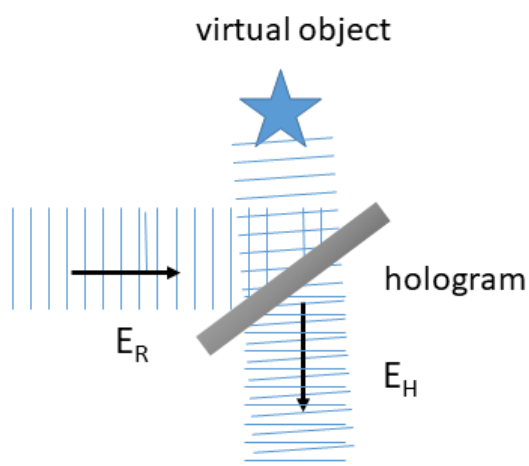

observer

b)

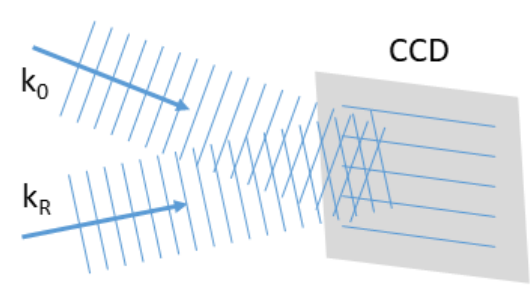

Fig. 1.3 a) Schematic of the holography method: hologram recording (left), and reconstruction (right). b) Object and reference wavefronts incident on the detector plane shifted by a small angle, in a configuration known as off-axis holography.

Mathematically, the reference and object waves, $E_{R}$ and $E_{O}$, are modelled by complex electric fields with amplitude $A$ and phase $\varphi$ :

$$
\begin{aligned}
& E_{R}(x, y, z)=A_{R}(x, y, z) \exp \left(i \varphi_{R}(x, y, z)\right) \\
& E_{O}(x, y, z)=A_{O}(x, y, z) \exp \left(i \varphi_{O}(x, y, z)\right)
\end{aligned}
$$

The recorded intensity at the detector plane $(z=0)$ is expressed as the square of the two complex fields:

$$
\begin{aligned}
I(x, y, 0)= & \left|E_{O}(x, y, 0)+E_{R}(x, y, 0)\right|^{2} \\
= & \left(E_{O}(x, y, 0)+E_{R}(x, y, 0)\right) \cdot\left(E_{O}(x, y, 0)+E_{R}(x, y, 0)\right)^{*} \\
= & E_{O}(x, y, 0) E_{O}^{*}(x, y, 0)+E_{R}(x, y, 0) E_{R}^{*}(x, y, 0) \\
& +E_{O}(x, y, 0) E_{R}^{*}(x, y, 0)+E_{R}(x, y, 0) E_{O}^{*}(x, y, 0)
\end{aligned}
$$

The first term is the intensity of the scattered light from the object $I_{O}(x, y, 0)=$ $E_{O}(x, y, 0) E_{O}^{*}(x, y, 0)$. The second term is the intensity of the reference wave $I_{R}(x, y, 0)=$ $E_{R}(x, y, 0) E_{R}^{*}(x, y, 0)$. Unlike the last two terms, these terms contain no information about the phase, and are therefore useless for the reconstruction process. 
6

The reconstruction procedure requires the illumination of the hologram with the reference wave. The resulting wave $E_{H}$ of the virtual image is:

$$
\begin{aligned}
E_{H}(x, y, z) \propto & E_{R}(x, y, z) \cdot I(x, y, 0) \\
E_{H}(x, y, z) \propto & E_{R}(x, y, z) \cdot\left(I_{R}(x, y, 0)+I_{O}(x, y, 0)\right) \\
& +E_{R}(x, y, z) \cdot\left(E_{O}(x, y, 0)+E_{R}^{*}(x, y, 0)\right) \\
& +E_{R}(x, y, z) \cdot\left(E_{R}(x, y, 0)+E_{O}^{*}(x, y, 0)\right)
\end{aligned}
$$

The first term is the zeroth diffraction order and corresponds to the reference wave. The second term is the +1 diffraction order and corresponds to the virtual image, or the wave diffracted by the object which we care about in this study. The third term is the -1 diffraction order and corresponds to a conjugate object image, called the real image, positioned symmetrically with respect to the virtual image.

One wishes to be able to select only the +1 order and suppress the other two. This is done experimentally by off-axis holography (Fig $1.3 \mathrm{~b}$ ), where the reference beam is shifted by a small angle with respect to the object beam [34]. As a consequence, the three diffraction orders are separated, allowing the selection of the desired order easily. These operations are usually performed in the Fourier space, or wave vectors k-space.

A fast Fourier transform (FFT) algorithm is used to reconstruct the original field. The hologram first undergoes a Fourier transform into the frequency space where spatial filtering of the unwanted diffraction orders takes place. The obtained complex field is then propagated towards the source before it is finally transformed back to the spatial domain. This is done by 4 main steps:

1. A first Fourier Transform to move to the frequency space:

$$
\widehat{E}_{H}\left(k_{x}, k_{y}, 0\right)=F T\left\{E_{H}(x, y, 0)\right\}
$$

2. Spatial Filtering in $\mathrm{k}$-space to select the +1 order and eliminate the other two:

$$
\hat{E}_{H}^{S F}\left(k_{x}, k_{y}, 0\right)=S F\left\{\hat{E}_{H}\left(k_{x}, k_{y}, 0\right)\right\}
$$

3. Propagation in $\mathrm{k}$-space from $\mathrm{z}=0$ to any plane $\mathrm{z}$. This is done by multiplying the resulting complex field by a z-propagation function $G\left(k_{x}, k_{y}, z\right)=\exp \left(i k_{z} z\right)$ :

$$
\hat{E}_{H}^{S F}\left(k_{x}, k_{y}, z\right)=\hat{E}_{H}^{S F}\left(k_{x}, k_{y}, 0\right) \cdot G\left(k_{x}, k_{y}, z\right)
$$

4. Second Fourier Transform to move back to the spatial domain:

$$
E_{O}(x, y, z)=F T^{-1}\left\{\hat{E}_{H}^{S F}\left(k_{x}, k_{y}, z\right)\right\}
$$

An example of the above reconstruction procedure is presented in Fig 1.4. Here, we use an aperture NSOM tip in contact with a sample made up of a $40 \mathrm{~nm}$ gold film on a glass substrate. A typical hologram recorded in the plane of the camera is shown in Fig 1.4 a. This hologram is the result of interference between the light scattered through the aperture probe and the reference beam. Because of our off-axis configuration, interference fringes can be clearly seen (Fig $1.4 \mathrm{a}$ inset). The first step of the reconstruction (Fourier transform of the recorded real space hologram) is shown in Fig 1.4 b, where we can clearly see the three diffraction orders separated in k-space. The zeroth order is seen at the center, and the +1 and -1 diffraction orders are separated symmetrically on both sides, also because of the off-axis configuration. The +1 interference term needed to reconstruct the real image (highlighted by a red circle) is chosen and the two other terms are filtered out. We then propagate the filtered Fourier transform of the 
hologram in k-space by multiplying by the propagator G. Finally, an inverse Fourier transform is performed which allows us to calculate the complex electromagnetic field in any z plane from the plane of the camera up to the plane of the NSOM tip. Piling up all the images together gives us the 3D reconstructed EM field scattered from the probe, where both the amplitude and phase can be calculated from a single hologram. Examples of the reconstructed images of intensity scattered through the substrate are shown in Fig $1.4 \mathrm{c}$ for $\mathrm{z}=10 \mu \mathrm{m}$ below the tip up to the contact position $(\mathrm{z}=0 \mu \mathrm{m})$.

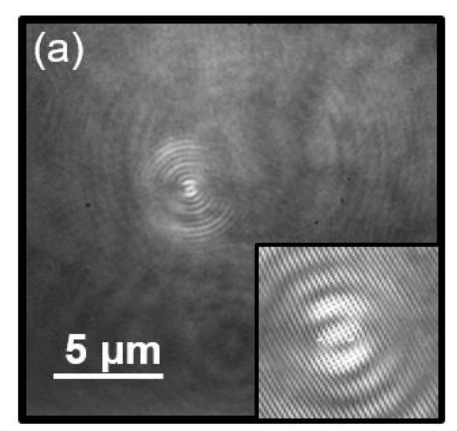

(c)
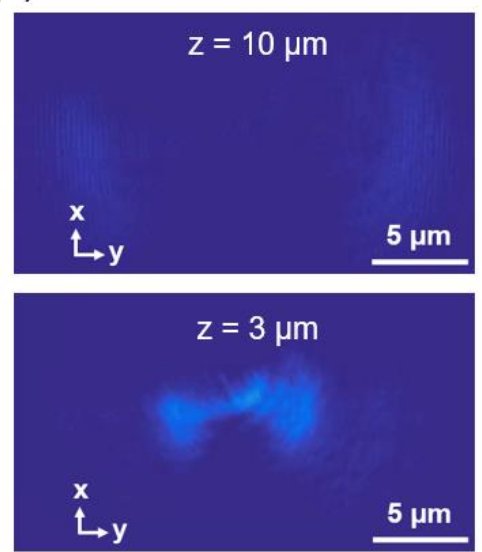
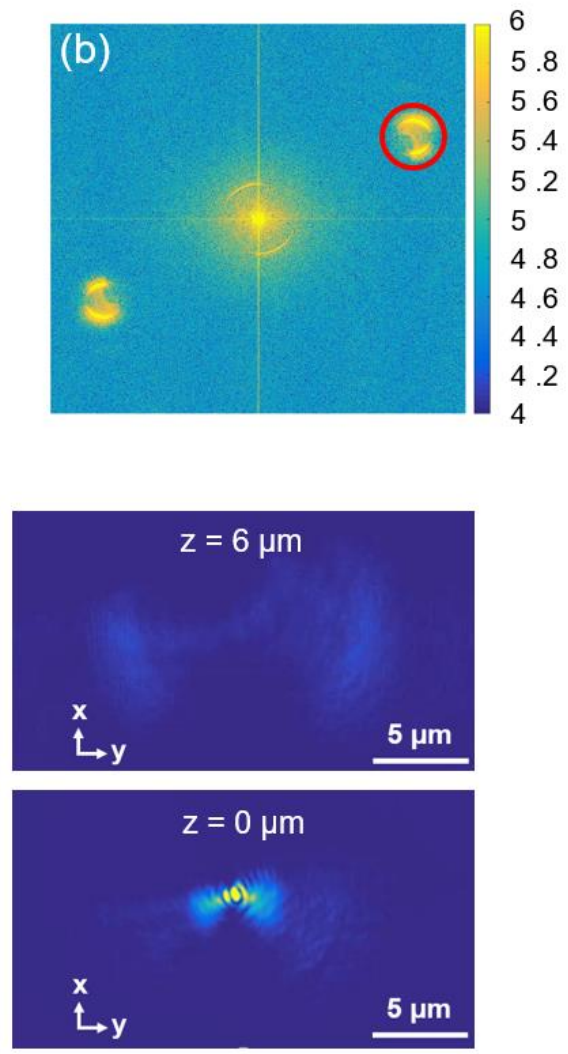

Fig. 1.4 a) Procedure for the reconstruction of the scattered EM field. a) Recorded hologram resulting from the interference between the reference beam and the light scattered by a NSOM probe through a plasmonic metal layer. Inset: magnified image showing the interference fringes. b) Fourier transform of the hologram showing the three diffraction orders in k-space: 0th order of diffraction in the center, +1 interference term (highlighted by the red circle, yields the real image after recontruction) and the -1 interference term (virtual image). c) Images of the field scattered by the tip in planes perpendicular to the tip axis, at distances varying between $\mathrm{z}=0$ and $\mathrm{z}=10 \mu \mathrm{m}$ from the tip.

\subsection{Near-field scanning optical microscopy combined with digital holography}

In this section, we describe the results that we obtained with our integrated holography-SNOM technique where we study the light scattered by nanosized probes in various environments. We observe that the aperture tip in free space scatters light mainly in the forward direction with a broad angular distribution. When it is placed in contact with a glass substrate, light is scattered 
8

exactly at an angle matching the critical angle of an air/glass interface. Finally, when the tip is placed in contact with a plasmonic metallic sample, leaky surface plasmons are observed at a supercritical emission angle. We also perform Finite Difference Time Domain (FDTD) simulations that support a model of the NSOM tip as a superposition of electric and magnetic dipoles.

\subsubsection{Experimental Setup}

In Fig. 1.5, we show a schematic of our optical setup, which is made up of a Mach-Zehnder off-axis interferometer combined with a commercial NSOM (WITec GmbH alpha 300s) [37] (figure taken from [38]). In our experiments we use a single mode $\mathrm{He}-\mathrm{Ne}$ laser (Research Electro Optics R-32413, $\lambda=633 \mathrm{~nm}, \mathrm{P}=35 \mathrm{~mW}$ ) that is coupled to a $90-10$ fiber splitter. This splits the incident light into a sample beam with $90 \%$ of the initial power and a reference beam with the remaining $10 \%$. We then focus our sample beam through a $20 \times, \mathrm{NA}=0.4$ objective at the apex of a $150 \mathrm{~nm} \mathrm{SiO} 2$ pyramidal aperture probe with an angle of $\sim 70^{\circ}$, coated with 120 $\mathrm{nm}$ of aluminum (Al). This tip is placed either in free space or in contact with the sample to be studied. A closer look at this type of probes is given in the optical and SEM images of Fig 1.6 (provided by the WITec company [39]). To finely control the relative position of the sample and the tip, we place our sample on a piezoelectric three-axis translation stage. Then the light transmitted through the tip apex is collected with a $100 \times, \mathrm{NA}=0.9$ objective when the tip is in free space, and a $100 \times, \mathrm{NA}=1.4$ oil immersion objective in the presence of a sample.

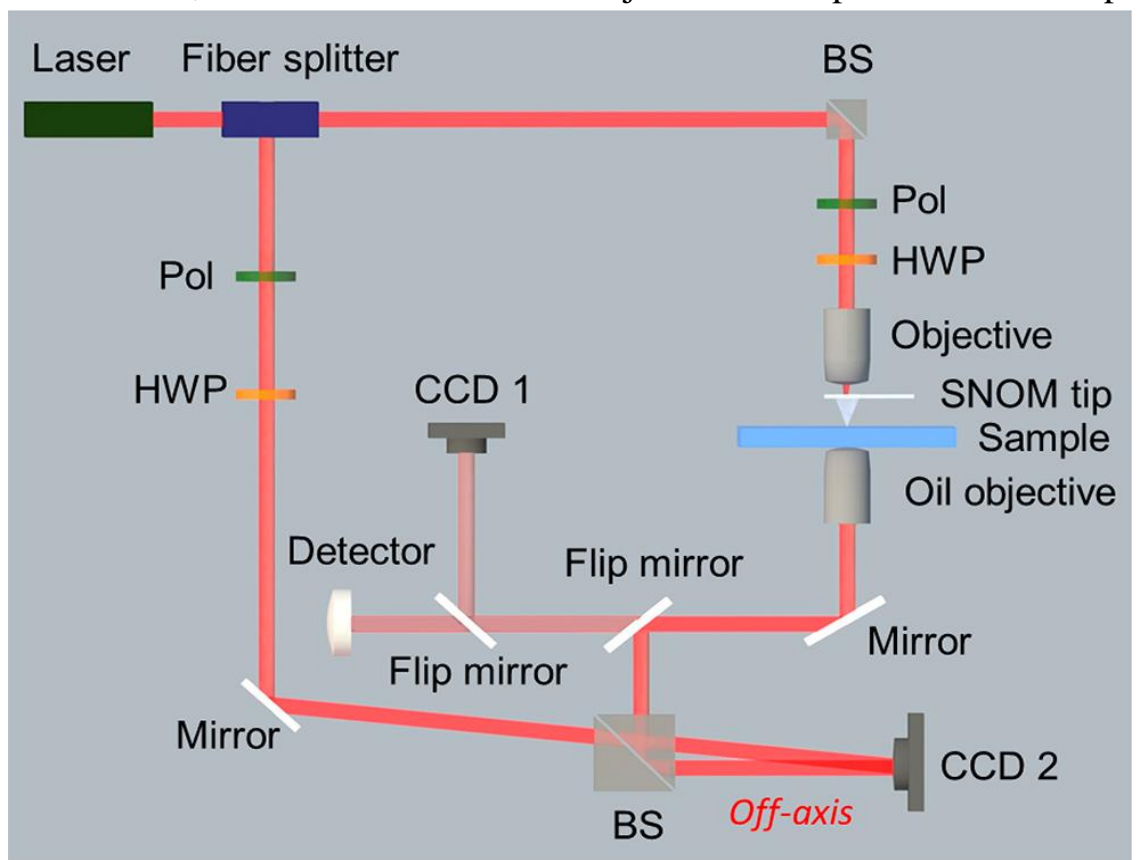

Fig. 1.5 Schematic of the optical setup: an off-axis digital holographic microscope (DHM) is combined with a near-field scanning optical microscope (NSOM) using a metal-coated hollow pyramidal aperture tip. BS: beam splitter, Pol: polarizer, HWP: half-wave plate, CCD: charge coupled device camera. Figure taken from [38]. 
It is then directed to a CMOS camera (Photon Focus MV-D1024 $\times 10-160-C L$, sensor resolution: $1024 \times 1024,8 \mu \mathrm{m} \times 8 \mu \mathrm{m}$ pixel matrix, $0.2 \mathrm{~s}$ exposure time). The reference beam is also sent with a few degree shift to the CMOS (off-axis configuration), where interference with the sample beam occurs. This detected hologram is all we need experimentally to be able to calculate the amplitude and phase of the scattered field and reconstruct the 3D pattern. Maximum contrast is attained by placing a polarizer and a half-wave plate in the path of both the sample and reference beams, ensuring that identical linear polarizations are being used. The reconstruction procedure is carried out numerically by a fast Fourier transform (FTT) algorithm following the procedure described in section 1.2.

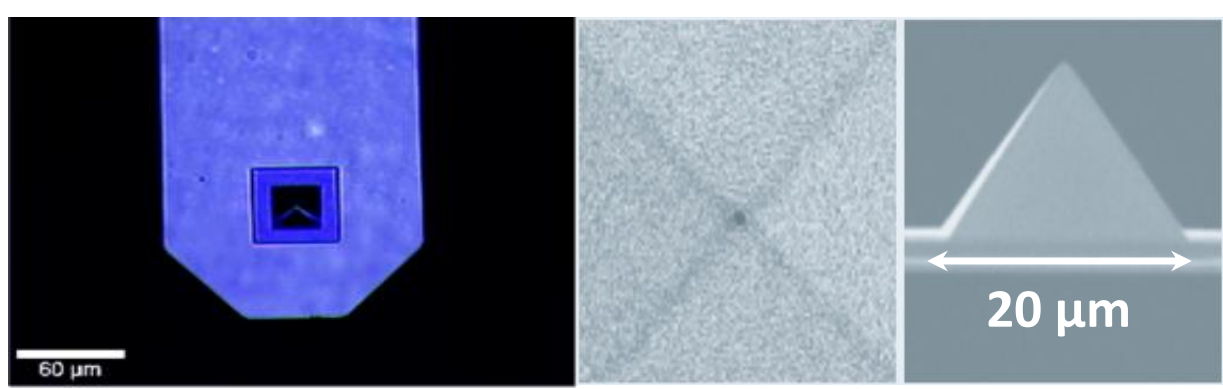

Fig. 1.6 Optical and SEM images of the NSOM probe with an aperture size $\sim 150 \mathrm{~nm}$. Images provided by the WITec company [39].

\subsubsection{D Reconstructed EM Field through Different Media}

With this method, we characterize the scattered radiation pattern of a hollow NSOM probe placed in free space, and coupled to two types of surfaces: a transparent glass sample made of a $160 \mu \mathrm{m}$ thick glass coverslip (VWR Micro Cover Glasses, No. 1), and a plasmonic sample made of a $40 \mathrm{~nm}$ gold film evaporated on an identical glass coverslip. The numerical analysis described in Fig 1.4 is adopted. It relies on the method proposed by Cuche et al. [25,34]. In our calculations, we approximate the nanoaperture as a point-like source, and thus account for the fast decay of the optical intensity as $\rho^{-2}$, where $\rho$ is the distance to the tip in spherical coordinates. Therefore, all the intensity graphs are multiplied by $\rho^{2}$ for clearer representation purposes.

We start by representing the reconstructed EM field for the probe placed in free-space (Fig. 1.7 a). A 2D cross section is taken in the $x^{-} z$ plane that is perpendicular to the sample surface $(\mathrm{x}-\mathrm{y})$, contains the axis of the linearly polarized illumination $(\mathrm{x})$, and is perpendicular to the axis of the cantilever (y). We chose to calculate the intensity (Fig. $1.7 \mathrm{~b}$ ), as well as the amplitude $|\mathrm{A}(\mathrm{x}, \mathrm{y}, \mathrm{z})|$ multiplied by the cosine of the corresponding phase $\cos \phi(\mathrm{x}, \mathrm{y}, \mathrm{z})$ (Fig. $1.7 \mathrm{c}$ ) which allows us to easily observe the wavefronts of the propagating field. A map of the phase alone can also be reconstructed with our method [34]. From these results, we infer that a tip placed in free-space, or in other words, without any coupling to an external environment, behaves as a Lambertian point source, scattering an EM field centered about $\theta=0^{\circ}$ with a broad angular distribution. In addition, Finite Difference Time Domain (FDTD) simulations were performed to compare these experimental results to the theoretical description. Those simulations were done using the Lumerical Solutions software, where our metal-coated aperture 
10

probe is modeled as a superposition of lateral magnetic $\left(\mathrm{M}_{\mathrm{y}} \propto \mathrm{H}_{\mathrm{y}}\right)$ and electric dipoles $\left(\mathrm{P}_{\mathrm{x}} \propto \mathrm{E}_{\mathrm{x}}\right)$ of respective strengths 2 and 1 , with $\mathrm{x}$ being the direction parallel to the incident light polarization direction. This model is adapted from the work of Obermüller and Karrai on the free space radiation of metal coated aperture tips [11]. In our simulations, we place a frequencydomain field monitor in the $x^{-} \mathrm{z}$ plane at the position of the NSOM tip, which allows us to calculate the complex EM intensity up to a distance of $10 \mu \mathrm{m}$ in the substrate. We place another monitor in the $\mathrm{x}-\mathrm{y}$ plane at a distance of $10 \mu \mathrm{m}$ below the sample surface, which determines using Fourier-transform calculations, the projection of the scattered EM field into the far field. We use perfectly matched layer (PML) absorbing boundary conditions (32 PML layers) that are impedance-matched to the simulation region and its materials, and the value of the complex permittivity of gold is $\varepsilon_{\text {gold }}=-12.047+1.163 i$ at $633 \mathrm{~nm}$, taken from Olmon et al [40]. It is important to note here that the simulations give only a qualitative approximation of the EM field radiated from the probes. This is due to two main reasons. First, this model can only be used to accurately describe small radiation angles [41], and second, the subwavelength details of the geometry of the metal-coated hollow probe and its interaction with two plane metal/dielectric interfaces are not taken into account [42]. The latter interaction can become important when the tip is coupled to a plasmonic sample [18,20]. We see from Fig $1.7 \mathrm{~d}$ that the simulation results are in good agreement with the experimental results. Note that this agreement is not achieved if the probe is modelled as an electric or magnetic dipole only, which confirms the fact that such hollow probes are best modelled as a superposition of perpendicular electric and magnetic dipoles.

Subsequently, we perform the same analysis for the tip placed in contact with the transparent glass sample (Fig 1.8), and then with the plasmonic gold film sample (Fig 1.9). For the glass sample, we observe that in addition to the forward scattering around $\theta=0^{\circ}$, light emerges along two preferred directions that correspond exactly to the critical angle of the airglass interface $\left(\left|\theta_{\text {c,glass }}\right|=41.8^{\circ}\right.$ from Snell's law). For the gold-coated sample, we observe that leaky surface plasmons are launched and emerge into the substrate at the resonance angles that satisfy the phase matching condition at the air-gold interface. This is calculated by the following conservation of momentum equation:

$$
n_{\text {glass }} \frac{2 \pi}{\lambda} \sin \theta_{c, \text { gold }}=\operatorname{Re}\left\{\frac{2 \pi}{\lambda} \sqrt{\frac{\varepsilon_{\text {gold }}}{\varepsilon_{\text {gold }}+1}}\right\}
$$

where $n_{\text {glass }}$ is the index of refraction of the glass substrate, $\lambda$ is the incident excitation wavelength, and $\varepsilon_{\text {gold }}$ is the complex permittivity of the gold film. Using the index of refraction of the glass coverslip provided by the manufacturer $\left(n_{\text {glass }}=1.525\right.$, from VWR International [43]), $\lambda=633 \mathrm{~nm}$, and $\varepsilon_{\text {gold }}=-12.047+1.163 i$ (from Olmon et al. [40]), we find that $\left|\theta_{\mathrm{c}, \text { gold }}\right|=$ $43.3^{\circ}$. We are able to detect such emission angles experimentally because of the use of an oil objective with a high numerical aperture. FDTD simulation results agree with the experimental results for both the transparent (Fig $1.8 \mathrm{~d}$ ) and the plasmonic sample (Fig $1.9 \mathrm{~d}$ ). 
(a)

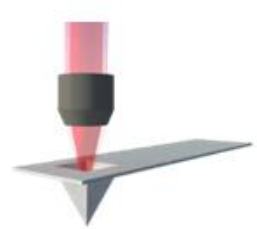

(c)

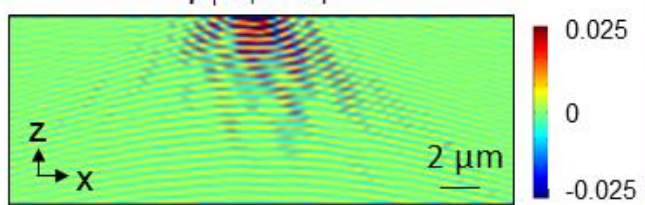

(b) $\quad \rho^{2} \mathrm{I}$

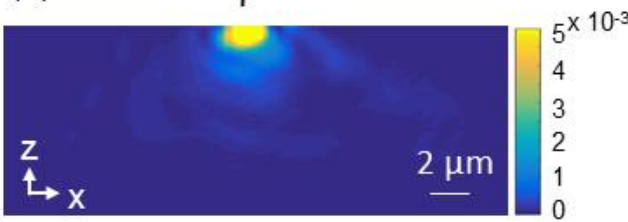

(d)

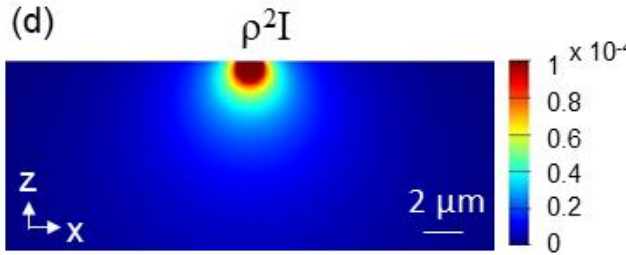

Fig. 1.7 Reconstructed EM field scattered from the hollow metal-coated aperture probe placed in air (free-space). b) Intensity profile in the x-z plane along the axis of the tip. For clarity, the intensity values are multiplied by $\rho^{2}$. c) Complex EM field represented by the product $\rho|\mathrm{A}(\mathrm{x}, \mathrm{y}, \mathrm{z})| \cos (\phi(\mathrm{x}, \mathrm{y}, \mathrm{z}))$, where $\mathrm{A}(\mathrm{x}, \mathrm{y}, \mathrm{z})$ is the amplitude, and $\phi(\mathrm{x}, \mathrm{y}, \mathrm{z}))$ the phase. Wavefronts are clearly observed. d) Corresponding FDTD simulated intensity multiplied by $\rho^{2}$.

(a)

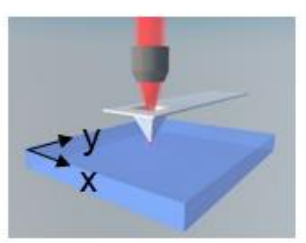

(c)

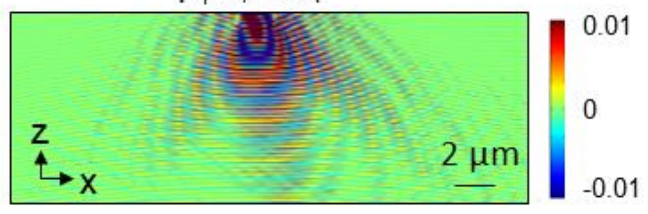

(b) $\quad \rho^{2} \mathrm{I}$

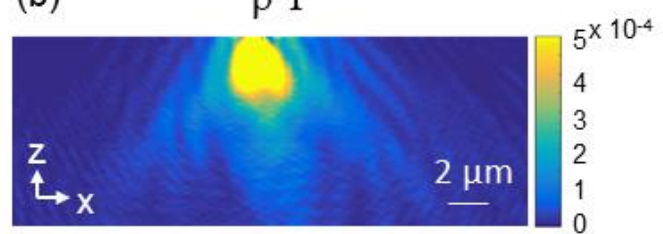

(d)

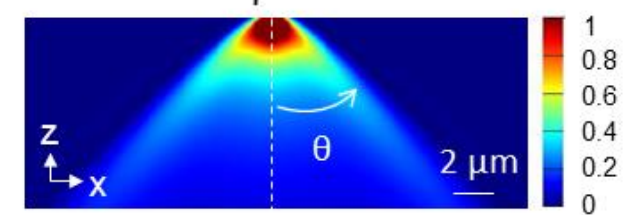

Fig. 1.8 Reconstructed EM field scattered from the hollow metal-coated aperture probe placed in contact with the transparent glass substrate. b) Intensity profile in the x-z plane along the axis of the tip. For clarity, the intensity values are multiplied by $\rho^{2}$. c) Complex EM field represented by the product $\rho|\mathrm{A}(\mathrm{x}, \mathrm{y}, \mathrm{z})| \cos (\phi(\mathrm{x}, \mathrm{y}, \mathrm{z}))$, where $\mathrm{A}(\mathrm{x}, \mathrm{y}, \mathrm{z})$ is the amplitude, and $\phi(\mathrm{x}, \mathrm{y}, \mathrm{z}))$ the phase. Wavefronts are clearly observed. d) Corresponding FDTD simulated intensity multiplied by $\rho^{2}$. 
12

(a)

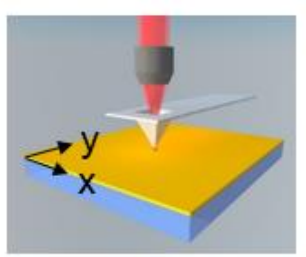

(c)

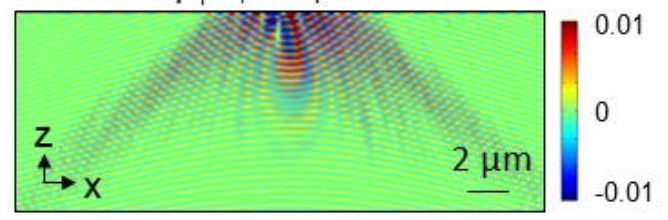

(b) $\quad \rho^{2} \mathrm{I}$

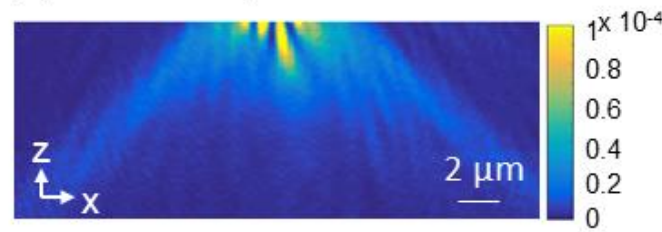

(d)

$\rho^{2} \mathrm{I}$

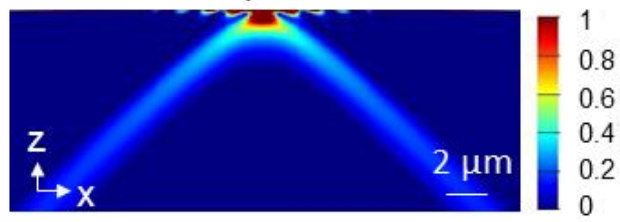

Fig. 1.9 Reconstructed EM field scattered from the hollow metal-coated aperture probe placed in contact with the plasmonic sample made of a gold thin film on a glass substrate. b) Intensity profile in the $x-z$ plane along the axis of the tip. For clarity, the intensity values are multiplied by $\rho^{2}$. c) Complex EM field represented by the product $\rho|A(x, y, z)| \cos (\phi(x, y, z))$, where $A(x, y, z)$ is the amplitude, and $\phi(x, y, z))$ the phase. Wavefronts are clearly observed. d) Corresponding FDTD simulated intensity multiplied by $\rho^{2}$.

The directional emission of the leaky surface plasmons observed at the two resonance angles in the presence of the gold film clarifies the presence of the two narrow lobes seen in the Fourier transform image of the hologram (Fig $1.4 \mathrm{~b}$ ). Because the probe is illuminated with linearly polarized light, surface plasmons are excited along a preferred direction which results in the two lobes observed both in k-space and in the reconstructed images in real space [17]. We also notice that due to the interference between the generated surface plasmons and the transmitted leaked radiation, fringes in the gold film are observed in both the experimental and simulation results (Fig $1.9 \mathrm{~b}$ and Fig 1.9d) [26]. This behavior is also seen in the $\mathrm{x}-\mathrm{y}$ intensity images presented in Fig 1.10 that show the two lobes of the surface plasmons excited by the incident linear illumination [6,17]. These results highly resemble the results obtained by Drezet el al. [18] that are given in Fig $1.10 \mathrm{~d}$. 


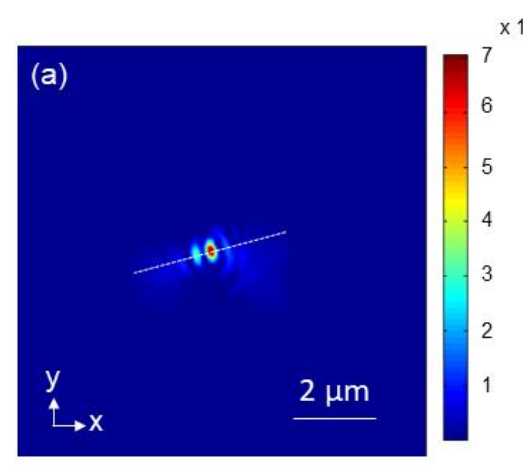

(b)
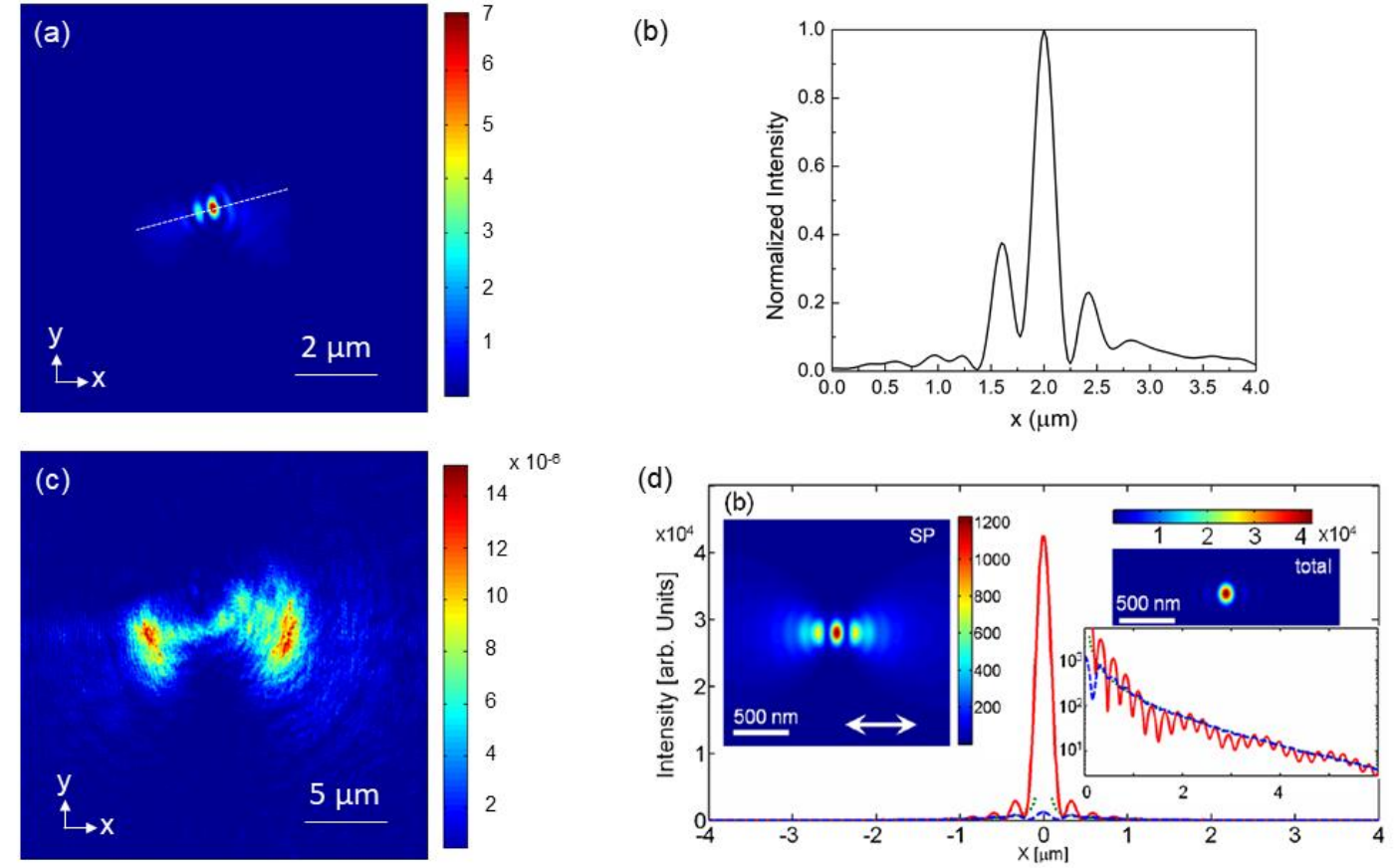

Fig. 1.10 Reconstructed intensity of the scattered EM field calculated in the $x-y$ plane at a) the surface of the metallic film sample, and at c) a distance of $5 \mu \mathrm{m}$ inside the substrate. b) Normalized intensity profile along the dashed line in a). d) Figure taken from Drezet et al. [18] for comparison: Direct space images associated with a point like dipole radiating through the metal film and recorded in the back-focal plane of the microscope ocular.

\subsubsection{Characterization of the Angular Scattering}

From the results presented in the previous section, we can quantify the angular radiation patterns of the light scattered by the NSOM tip through different environments. In the graphs of Fig 1.11, we plot the normalized intensity of the scattered light as a function of the emission angle in polar coordinates for the three cases studied previously; tip in free-space (Fig 1.11 a), in contact with the glass substrate (Fig $1.11 \mathrm{~b}$ ), and in contact with the gold film on a glass substrate (Fig $1.11 \mathrm{c}$ ). The plotted experimental data points (in blue) are in spherical coordinates and are selected in a way to obey two selection rules. First, they are chosen to lie in the $\mathrm{x}-\mathrm{z}$ plane of the NSOM tip perpendicular to the surface of the sample. Second, we made sure that each angle $\theta$ has only one attributed intensity value. To this end, we select the data that lie only in a spherical shell of thickness $\Delta \rho=30 \mathrm{~nm}$ centered on the aperture tip. The corresponding FDTD simulation results of the far-field radiation patterns of a superposition of a magnetic and electric dipole are plotted in black. Very good agreement is obtained for all the three cases. The angular radiation patterns confirm the observations presented in section 1.3.2 (Figures 1.7, 1.8 and 1.9). In fact, we observe that the intensity of the scattered light from the tip in free-space decays exponentially around a maximum value centered at $\theta=0^{\circ}$ (Fig $1.11 \mathrm{~d}$ ). We also 
14

quantitatively verify that for the tip placed in contact with the glass sample (Fig $1.11 \mathrm{e}$ ), most of the light is scattered exactly at the critical angle $\pm\left|\theta_{\mathrm{c}, \text { glass }}\right|=41.8^{\circ}$ in addition to a broad distribution around $\theta=0^{\circ}$. As for the plasmonic sample, we verify that the generated leaky plasmons are transmitted into the substrate at an angle of $\pm\left|\theta_{c, \text { gold }}\right|=43.3^{\circ}$, which is higher than the critical angle of the medium. This highly directional scattering, narrower than in the previous cases, again reinforces our previous observation of the two lobes with a preferential direction caused by the surface plasmons. Surface plasmons are indeed highly selective in angle, both for their excitation and, as shown here, their leakage.

(a)

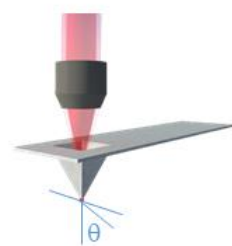

(d)

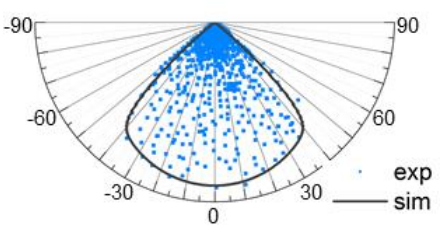

(b)

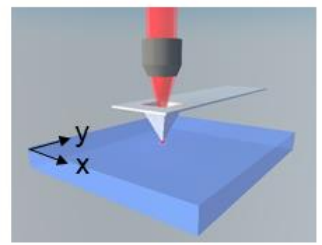

(e)

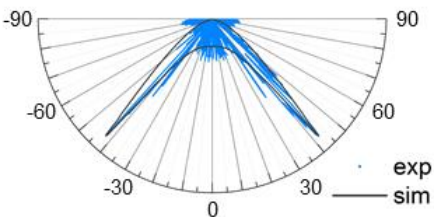

(c)

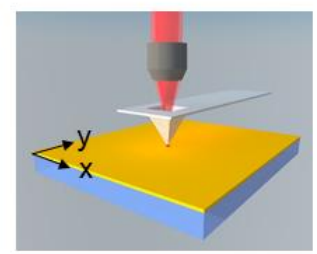

(f)

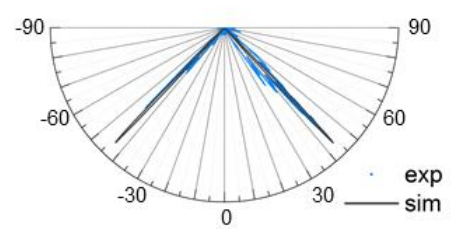

Fig. 1.11 Angular radiation patterns of the scattered light from a NSOM probe placed in a) air, b) in contact with a glass substrate, and c) a gold thin film on a glass substrate. d), e), f) Normalized intensity plotted in polar coordinates as a function of the angle of emission $\theta$. Emission peaks point to an angle $\pm\left|\theta_{\mathrm{c}, \text { glass }}\right|=41.8^{\circ}$ in e) and to $\pm\left|\theta_{\mathrm{c}, \text { gold }}\right|=43.3^{\circ}$ in f).

Next, we aim at comparing the behavior of the NSOM probe in the case where it is placed in the far-field region above the sample to the case where it is placed in contact with the sample in the near-field region. To do that, we place the tip at a distance of $3 \mu \mathrm{m}$ above the sample surface, and repeat the same characterization procedure. The angular radiation pattern is plotted in Fig 1.12. By comparing Fig 1.12 b to Fig 1.11 e, we notice the very high resemblance between the two plots, which allows us to conclude that the gold film has no effect on the transmitted light through the substrate when the incident light source is placed in the far field. This is because in this case, only small wavevectors are created by the tip, and therefore surface plasmons cannot be generated. The angular emission thus corresponds to that of a glass substrate, with maxima occurring at an angle of $\pm 40.5^{\circ}$ (below $\theta_{\text {c,glass }}$ ). We verified this result by performing the same experiment with the tip placed at a $3 \mu \mathrm{m}$ height above a glass substrate, and the same exact emission angle was observed. In addition, due to the weak transmission of the gold film, the maximum intensity at $\rho=10 \mu \mathrm{m}$ is found to decrease by a factor of 2.5 when the tip is placed in the far field. These results agree with those obtained by Hecht et al. [17] using back-focal plane and real space imaging. The complex EM field represented by the product $\rho|\mathrm{A}(\mathrm{x}, \mathrm{y}, \mathrm{z})| \cos (\phi(\mathrm{x}, \mathrm{y}, \mathrm{z}))$ is shown in Fig $1.12 \mathrm{c}$, where we can also clearly see that there are no leaky surface plasmons generated as opposed to the case where the tip is in contact with the film (Fig $1.9 \mathrm{c}$ ). 
(a)

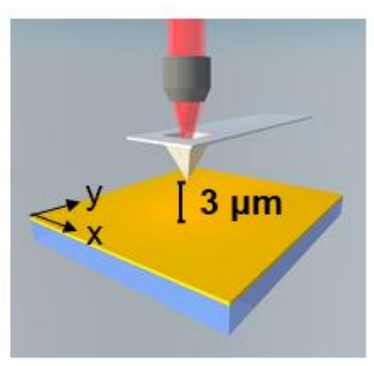

(b)

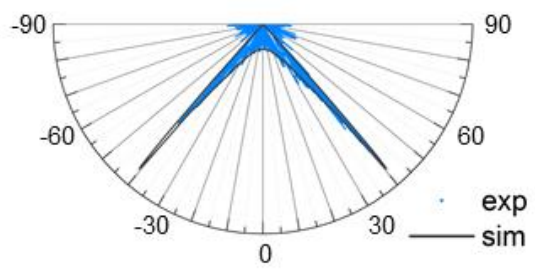

(c)

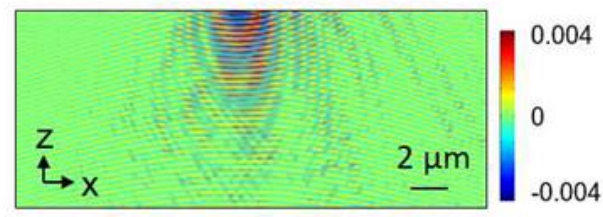

Fig. 1.12 Angular radiation patterns of the scattered light from a NSOM probe placed a) at 3 $\mu \mathrm{m}$ above a gold thin film on a glass substrate. b) Normalized intensity plotted in polar coordinates as a function of the angle of emission $\theta$. Emission peaks point to an angle $\pm|\theta|=$ $40.5^{\circ}$. c) Corresponding complex EM field represented by the product $\rho|A(x, y, z)| \cos (\phi(x, y, z))$, where $\mathrm{A}(\mathrm{x}, \mathrm{y}, \mathrm{z})$ is the amplitude, and $\phi(\mathrm{x}, \mathrm{y}, \mathrm{z}))$ the phase.

\subsection{Possible Applications}

\subsubsection{Coupled Nanoantennas}

Now that we are certain of the functionality of our near-field scanning optical microscope combined with digital holography, we can use it to fully characterize the scattered electromagnetic field from any nanostructure. Optical nanoantennas are interesting candidates due to their unique control of absorption and emission at the nanometer scale: high confinement, enhancement and directivity of electromagnetic radiation at a subwavelength dimensions. They have wide applications such as wavelength tuning, nano-trapping, nano-sensing, near-field imaging, photodetection, directional emission, etc. As discussed above, many techniques such as back-focal plane imaging were successfully used to describe the radiation pattern and scattering angles of different types of nanoantennas. However, we emphasize that the advantage of our technique is that both the amplitude and phase of the EM field scattered by an optical nanoantenna can be measured in one plane located in the far field, and then back-propagated to perform 3D reconstructions near the nano-antenna. Previous work using heterodyne holography were successfully done by the group of G. Tessier, where the simultaneous localization and selection of gold nanoparticles in three dimensions was studied with near-diffraction resolution [44]. The full scattered three-dimensional electromagnetic field of plasmonic gold nanodisk chains fabricated by e-beam lithography was also measured using the same holography technique [25] using single-shot hologram acquisition associated to a reconstruction of the 3D scattering pattern of the antennas. Cross sections of the reconstructed scattered field in different 
16

planes is shown in Fig 1.13 (taken from the paper of Suck et al. [25]). Although the holographic images are diffraction-limited, the shape of the disk chain is clearly identified, as well as electromagnetic hotspots that are created in the nanoantennas. In addition, strong directional scattering is observed (note the different scalebars in 1.13a and 1.13b, showing an approximately x6 enhancement of the scattering near the resonant wavelength).

With the combined NSOM-holography setup described in the present chapter, such studies can be extended further to study the coupling between NSOM tips and different types of optical nanoantennas. The full 3D scattering pattern of a locally excited nanoantenna and the influence of the position of the excitation source should become accessible. As mentioned earlier, recent studies have shown that a metal-coated hollow pyramidal probe coupled to a nanoantenna behaves as a tangential magnetic dipole. A schematic of the mentioned results is shown in Fig 1.14 and is taken from the paper of Denkova et al. [14]. Here, digital holography can be helpful to experimentally characterize this coupling behavior between nanosized probes and different types of nanoantennas using only a single hologram.

Another application would be to characterize the scattering behavior of NSOM probes with embedded nanoantennas at their extremities $[23,45]$. Due to the presence of defects at the subwavelength scale [46], the optical response of such nanoantennas might strongly deviate from expectations, and will depend on the environment. This problem can be fully addressed and controlled by our technique as well.
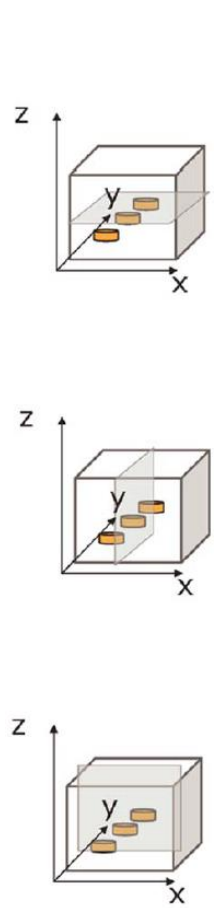

(a)

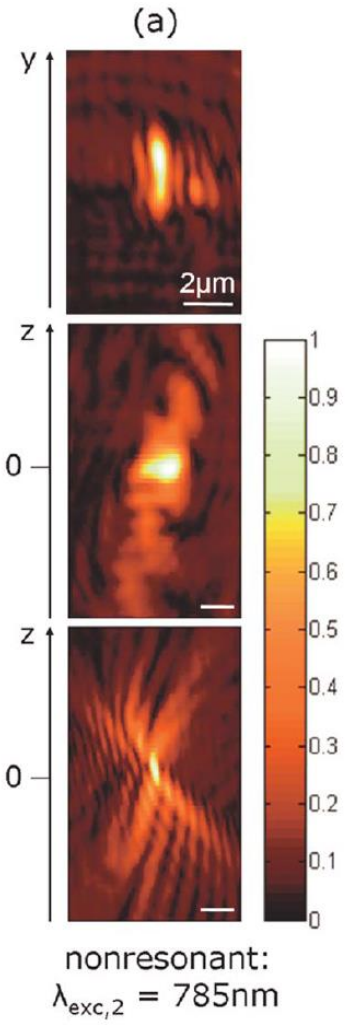

(b)

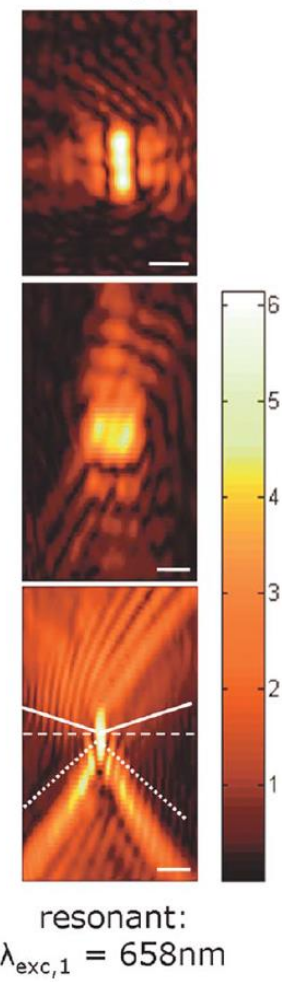

Fig. 1.13 Scattered field reconstructed from a single hologram of a nanodisk chain at nonresonant $(\lambda=785 \mathrm{~nm})(\mathrm{a})$ and resonant $(\lambda=658 \mathrm{~nm})(\mathrm{b})$ wavelengths, for an illumination polarized along the long axis (y) of the nanoantenna system. Cross-sections along different planes centered on the antenna: $\mathrm{x}-\mathrm{y}$ (top), $\mathrm{y}-\mathrm{z}$ (middle) and $\mathrm{x}-\mathrm{z}$ (bottom). Figure taken from [25]. 

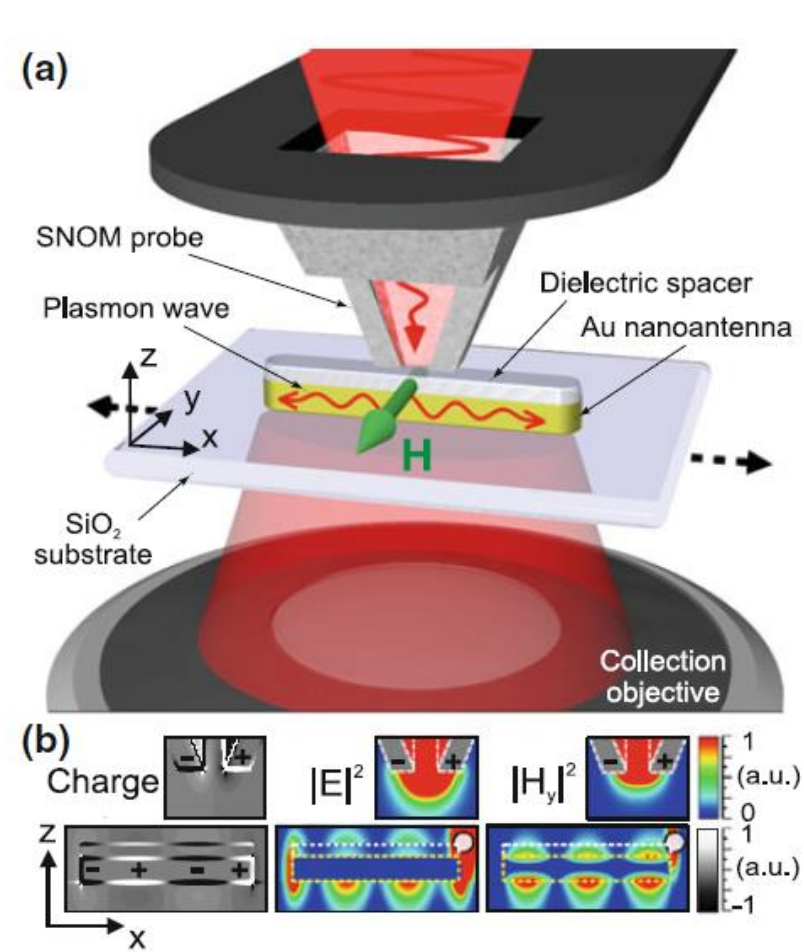

Fig. 1.14 a) Schematic of a hollow-pyramid NSOM probe coupled to a plasmonic nanoantenna. b) Simulations of the charge density, electric and magnetic field distributions. Figure taken from [14].

\subsubsection{Brownian nanoparticles as stochastic optical probes}

In much the same way as a scattering NSOM probe, nanoparticles can be used to scatter the local near field. In liquids, one can take advantage of the Brownian motion of nanoparticles in order to explore the volume of the sample: the movement of the probes is then stochastic, instead of deterministic as in classical NSOM experiments. Each of the particles can behave as a subwavelength probe and, as it moves in an illuminated region of the sample, scatter the local field towards the holographic microscope described above. The reconstruction of the scattering field allows the 3D super localization of the particle, i.e. the determination of its center of mass with an accuracy which is only limited by the signal-to-noise ratio of the detection [47]. This localization can be achieved with $3 \times 3 \times 10 \mathrm{~nm}^{3}$ accuracy, and the scattering intensity is directly proportional to the local optical field provided that the particles are monodisperse, and therefore have identical scattering cross sections. Fig.1.15 shows the 3D image of a focused laser beam obtained by accumulating 36000 localization events. As shown in [48], the acquisition time required to reach the desired volume coverage can be estimated, and a full superresolved 3D image of the optical scene can be acquired with a resolution which is only limited by either the size of the particles (here, $100 \mathrm{~nm}$ ) or the localization accuracy. 


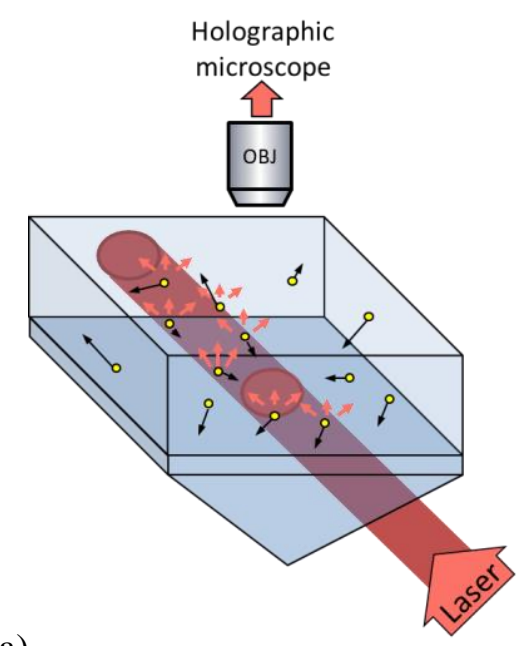

a)

Fig. 1.15 a) schematic description of an experiment using gold nanoparticles in Brownian motion in water as local probes. A $\lambda=660 \mathrm{~nm}$ diode laser beam is focused in water. Light is scattered by $\mathrm{r}=50 \mathrm{~nm}$ gold nanoparticles towards a holographic microscope. Each particle is localized in 3D with a $3 \times 3 \times 10 \mathrm{~nm}^{3}$ accuracy in post-processing. b) 3D position of 36000 localization events. The intensity $\mathrm{I}(\mathrm{x}, \mathrm{y}, \mathrm{z})$ recorded at each location is represented by the size of the spheres. The resolution of the image is limited only by the localization accuracy and the size of the particle, both well below the diffraction limit. Adapted from [47].

\subsubsection{Scattering through Disordered Media}

It has been recently proposed that scattering media can be used to couple the information contained in the near-field wave vectors to the observable far-field with conventional optics [49]. The multiple elastic scattering that light experiences in such a medium exhibits timereversal symmetry, a property that has been used in optics to achieve image transmission through opaque materials [50] or perfect absorption [51], among other phenomena.

Near-field scanning optical microscope combined with digital holography can be applied to probe the optical properties of such strongly scattering media using aperture tips. The nanosized tips act as point-like sources and are used to excite the input modes of strongly scattering media. The transmitted far-field signal is measured holographically and, as shown before, the complex field can be reconstructed at any point in space between the camera and the output of the scattering media. This allows us to accurately describe the amplitude and phase of the propagating electromagnetic field and compare its behavior through different scattering media. An example of holographic reconstruction of the 3D speckle formed after placing a NSOM tip acting as a temporally coherent nanolight source on a disorderd scattering medium made of $100 \mathrm{~nm}$ diameter TiO2 nanoparticles is shown in Fig. 1.16. This reconstruction can be seen as the spatial point spread function corresponding to a local sub- $\lambda$ sized excitation of the disordered medium. By measuring a hologram when the NSOM tip is at every possible location over the disordered medium, the full spatial response of the medium (or its Green's function) can be determined. Conversely, this can subsequently be used as a superlens allowing superresolution imaging in the visible spectrum. With such holographic characterization combined to point-like excitation of the disordered medium, it therefore becomes possible to get access to 
full-field subwavelength imaging of structures containing high spatial frequencies typically not propagating into the far-field. A proof of principle of the coupling of the near-field information to the far field by means of a disordered medium, and of its holographic detection has been achieved by Park et al. [49]. Fig. 1.17, taken from the cited paper by Park et al., shows a schematic of the experimental concept where the transmitted far-field signal generated from a near-field aperture tip has been detected holographically at different locations on the sample, and the original field has been recovered.
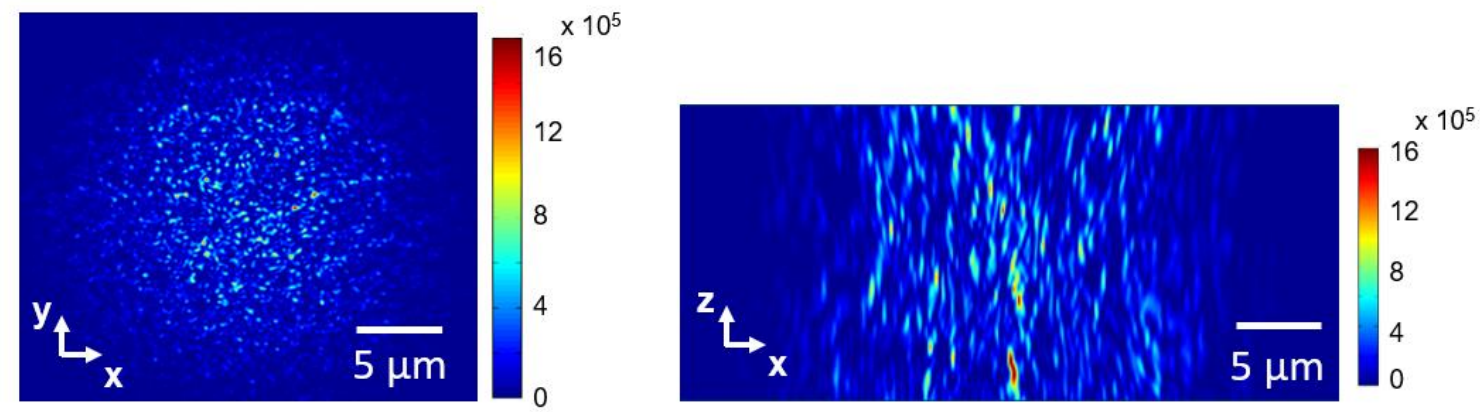

Fig. 1.16 Intensity plots in the x-y plane (left) and x-z plane (right) of the reconstructed speckle generated by the scattered light from the NSOM tip through a disordered medium made up of $100 \mathrm{~nm} \mathrm{TiO} 2$ nanoparticles.

(a)

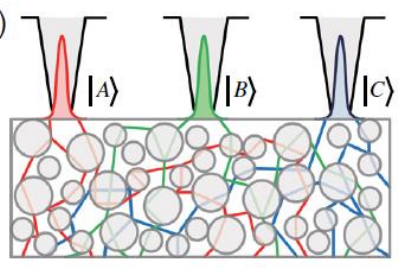

varyma

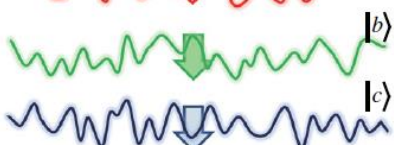

(b)
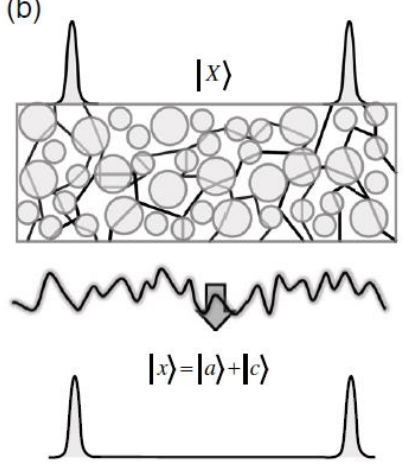

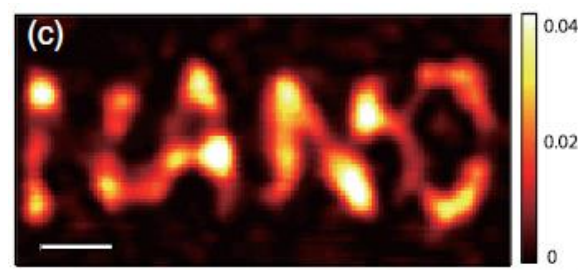
. 
20

electromagnetic field from a subwavelength probe. This is possible because, from a single recorded hologram, we are able to obtain information not only about the intensity, but also about the phase of the scattered electromagnetic field in any plane away from the tip. We applied this method to fully characterize the light scattered by the metal-coated hollow pyramidal aperture tip of a NSOM placed in free space, and coupled to transparent and plasmonic media. We supported our experimental results with FDTD simulations that model such type of probes as a superposition of an electric and magnetic dipole. We can also conclude that the behavior of a NSOM probe is highly related to its coupling to the environment.

This experimental and numerical validation opens the way for a broad range of new applications taking advantage of both the subwavelength localization possibilities of SNOM and the amplitude and phase imaging capabilities of holography. The study of complex subwavelength systems such as nanoantennas and resonators, as well as multiple scattering through disordered media should now be within reach.

\section{References}

1. D. W. Pohl, W. Denk, and M. Lanz, "Optical stethoscopy: Image recording with resolution 入/20," Appl. Phys. Lett. 44(7), 651-653 (1984).

2. D. Costantini, L. Greusard, A. Bousseksou, R. Rungsawang, T. P. Zhang, S. Callard, J. Decobert, F. Lelarge, G.-H. Duan, Y. De Wilde, and R. Colombelli, "In Situ Generation of Surface Plasmon Polaritons Using a Near-Infrared Laser Diode," Nano Lett. 12(9), 46934697 (2012).

3. E. H. Synge, "A suggested method for extending microscopic resolution into the ultramicroscopic region," Lond. Edinb. Dublin Philos. Mag. J. Sci. 6(35), 356-362 (1928).

4. E. H. Synge, "An application of piezo-electricity to microscopy," Lond. Edinb. Dublin Philos. Mag. J. Sci. 13(83), 297-300 (1932).

5. U. Dürig, D. W. Pohl, and F. Rohner, "Near-field optical-scanning microscopy," J. Appl. Phys. 59(10), 3318-3327 (1986).

6. L. Novotny and B. Hecht, "Principles of Nano-Optics", Cambridge University Press (2006).

7. E. J. Sánchez, L. Novotny, and X. S. Xie, "Near-field fluorescence microscopy based on two-photon excitation with metal tips," Phys. Rev. Lett. 82(20), 4014 (1999).

8. J.-J. Greffet and R. Carminati, "Image formation in near-field optics," Prog. Surf. Sci. 56(3), 133-237 (1997).

9. D. Costantini, A. Bousseksou, M. Fevrier, B. Dagens, and R. Colombelli, "Loss and gain measurements of tensile-strained quantum well diode lasers for plasmonic devices at telecom wavelengths," IEEE J. Quantum Electron. 48(1), 73-78 (2012).

10. H. A. Bethe, "Theory of diffraction by small holes," Phys. Rev. 66(7-8), 163 (1944).

11. C. Obermüller and K. Karrai, "Far field characterization of diffracting circular apertures," Appl. Phys. Lett. 67(23), 3408-3410 (1995).

12. C. Obermüller, K. Karrai, G. Kolb, and G. Abstreiter, "Transmitted radiation through a subwavelength-sized tapered optical fiber tip," Ultramicroscopy 61(1), 171-177 (1995).

13. A. Drezet, J. C. Woehl, and S. Huant, "Diffraction by a small aperture in conical geometry: Application to metal-coated tips used in near-field scanning optical microscopy," Phys. Rev. E 65(4), (2002).

14. D. Denkova, N. Verellen, A. V. Silhanek, V. K. Valev, P. V. Dorpe, and V. V. Moshchalkov, "Mapping Magnetic Near-Field Distributions of Plasmonic Nanoantennas," ACS Nano 7(4), 3168-3176 (2013). 
15. A. Babuty, K. Joulain, P.-O. Chapuis, J.-J. Greffet, and Y. De Wilde, "Blackbody Spectrum Revisited in the Near Field," Phys. Rev. Lett. 110(14), 146103 (2013).

16. K. Joulain, P. Ben-Abdallah, P.-O. Chapuis, Y. De Wilde, A. Babuty, and C. Henkel, "Strong tip-sample coupling in thermal radiation scanning tunneling microscopy," J. Quant. Spectrosc. Radiat. Transf. 136(Supplement C), 1-15 (2014).

17. B. Hecht, H. Bielefeldt, L. Novotny, Y. Inouye, and D. W. Pohl, "Local Excitation, Scattering, and Interference of Surface Plasmons," Phys. Rev. Lett. 77(9), 1889-1892 (1996).

18. A. Drezet and C. Genet, "Imaging Surface Plasmons: From Leaky Waves to Far-Field Radiation," Phys. Rev. Lett. 110(21), 213901 (2013).

19. P. Bharadwaj, A. Bouhelier, and L. Novotny, "Electrical Excitation of Surface Plasmons," Phys. Rev. Lett. 106(22), (2011).

20. R. Marty, C. Girard, A. Arbouet, and G. Colas des Francs, "Near-field coupling of a pointlike dipolar source with a thin metallic film: Implication for STM plasmon excitations," Chem. Phys. Lett. 532, 100-105 (2012).

21. A. Drezet, A. Hohenau, D. Koller, A. Stepanov, H. Ditlbacher, B. Steinberger, F. R. Aussenegg, A. Leitner, and J. R. Krenn, "Leakage radiation microscopy of surface plasmon polaritons," Mater. Sci. Eng. B 149(3), 220-229 (2008).

22. M. Berthel, Q. Jiang, A. Pham, J. Bellessa, C. Genet, S. Huant, and A. Drezet, "Directional Local Density of States of Classical and Quantum Propagating Surface Plasmons," Phys. Rev. Appl. 7(1), 14021 (2017).

23. A. G. Curto, G. Volpe, T. H. Taminiau, M. P. Kreuzer, R. Quidant, and N. F. van Hulst, "Unidirectional Emission of a Quantum Dot Coupled to a Nanoantenna," Science 329(5994), 930-933 (2010).

24. M. A. Lieb, J. M. Zavislan, and L. Novotny, "Single-molecule orientations determined by direct emission pattern imaging," JOSA B 21(6), 1210-1215 (2004).

25. S. Y. Suck, S. Collin, N. Bardou, Y. De Wilde, and G. Tessier, "Imaging the threedimensional scattering pattern of plasmonic nanodisk chains by digital heterodyne holography," Opt. Lett. 36(6), 849-851 (2011).

26. C. Park, J.-H. Park, C. Rodriguez, H. Yu, M. Kim, K. Jin, S. Han, J. Shin, S. H. Ko, K. T. Nam, Y.-H. Lee, Y.-H. Cho, and Y. Park, "Full-Field Subwavelength Imaging Using a Scattering Superlens," Phys. Rev. Lett. 113(11), (2014).

27. D. Gabor, A New Microscopic Principle (Nature Publishing Group, 1948).

28. D. Gabor, "Microscopy by reconstructed wave-fronts," Proc R Soc Lond A 197(1051), 454 487 (1949).

29. E. N. Leith and J. Upatnieks, "Reconstructed wavefronts and communication theory," JOSA 52(10), 1123-1130 (1962).

30. U. Schnars and W. P. Jüptner, "Digital recording and numerical reconstruction of holograms," Meas. Sci. Technol. 13(9), R85 (2002).

31. J. W. Goodman and R. W. Lawrence, "Digital image formation from electronically detected holograms," Appl. Phys. Lett. 11(3), 77-79 (1967).

32. M. A. Kronrod, N. S. Merzlyakov, and L. P. Yaroslavskii, "Reconstruction of a hologram with a computer," Sov. Phys. Tech. Phys. 17, 333 (1972).

33. U. Schnars and W. Jüptner, "Direct recording of holograms by a CCD target and numerical reconstruction," Appl. Opt. 33(2), 179-181 (1994).

34. E. Cuche, P. Marquet, and C. Depeursinge, "Spatial filtering for zero-order and twin-image elimination in digital off-axis holography," Appl. Opt. 39(23), 4070-4075 (2000).

35. F. Le Clerc, L. Collot, and M. Gross, "Numerical heterodyne holography with twodimensional photodetector arrays," Opt. Lett. 25(10), 716-718 (2000).

36. M. Gross and M. Atlan, "Digital holography with ultimate sensitivity," Opt. Lett. 32(8), 909-911 (2007). 
22

37. V. Parigi, E. Perros, G. Binard, C. Bourdillon, A. Maître, R. Carminati, V. Krachmalnicoff, and Y. D. Wilde, "Near-field to far-field characterization of speckle patterns generated by disordered nanomaterials," Opt. Express 24(7), 7019-7027 (2016).

38. N. Rahbany, I. Izeddin, V. Krachmalnicoff, R. Carminati, G. Tessier, and Y. De Wilde, "One-Shot Measurement of the Three-Dimensional Electromagnetic Field Scattered by a Subwavelength Aperture Tip Coupled to the Environment," ACS Photonics 5(4), 15391545 (2018).

39. www.witec.de, "Witec GmbH".

40. R. L. Olmon, B. Slovick, T. W. Johnson, D. Shelton, S.-H. Oh, G. D. Boreman, and M. B. Raschke, "Optical dielectric function of gold," Phys. Rev. B 86(23), 235147 (2012).

41. A. Drezet, J. C. Woehl, and S. Huant, "Extension of Bethe's diffraction model to conical geometry: Application to near-field optics," EPL Europhys. Lett. 54(6), 736 (2001).

42. A. Drezet, A. Cuche, and S. Huant, "Near-field microscopy with a single-photon point-like emitter: Resolution versus the aperture tip?," Opt. Commun. 284(5), 1444-1450 (2011).

43. www.vwr.com, "VWR International - Chemicals and Laboratory Scientific Supplies".

44. E. Absil, G. Tessier, M. Gross, M. Atlan, N. Warnasooriya, S. Suck, M. Coppey-Moisan, and D. Fournier, "Photothermal heterodyne holography of gold nanoparticles," Opt. Express 18(2), 780-786 (2010).

45. Z. Xie, Y. Lefier, M. A. Suarez, M. Mivelle, R. Salut, J.-M. Merolla, and T. Grosjean, "Doubly Resonant Photonic Antenna for Single Infrared Quantum Dot Imaging at Telecommunication Wavelengths," Nano Lett. 17(4), 2152-2158 (2017).

46. M. Thomas, J. J. Greffet, R. Carminati, and J. R. Arias-Gonzalez, "Single-molecule spontaneous emission close to absorbing nanostructures," Appl. Phys. Lett. 85(17), 38633865 (2004).

47. A. Martinez-Marrades, J.-F. Rupprecht, M. Gross, and G. Tessier, "Stochastic 3D optical mapping by holographic localization of Brownian scatterers," Opt. Express 22(23), 2919129203 (2014).

48. J.-F. Rupprecht, A. Martinez-Marrades, Z. Zhang, R. Changede, P. Kanchanawong, and G. Tessier, "Trade-offs between structural integrity and acquisition time in stochastic superresolution microscopy techniques," Opt. Express 25(19), 23146-23163 (2017).

49. C. Park, J.-H. Park, C. Rodriguez, H. Yu, M. Kim, K. Jin, S. Han, J. Shin, S. H. Ko, K. T. Nam, Y.-H. Lee, Y.-H. Cho, and Y. Park, "Full-Field Subwavelength Imaging Using a Scattering Superlens," Phys. Rev. Lett. 113(11), 113901 (2014).

50. S. M. Popoff, G. Lerosey, M. Fink, A. C. Boccara, and S. Gigan, "Image Transmission Through an Opaque Material," Nat. Commun. 1(6), 1-5 (2010).

51. W. Wan, Y. Chong, L. Ge, H. Noh, A. D. Stone, and H. Cao, "Time-Reversed Lasing and Interferometric Control of Absorption," Science 331(6019), 889-892 (2011). 